\title{
Association between low thyroid-stimulating hormone, posterior cortical atrophy and nitro-oxidative stress in elderly patients with cognitive dysfunction
}

\author{
Adriana E. Bulboacă로 Sorana D. Bolboacă², Angelo C. Bulboacă³ , Călin I. Prodan ${ }^{4}$
}

\author{
1Department of Pathophysiology, Iuliu Hațieganu University of Medicine and \\ Pharmacy, Cluj-Napoca, Romania \\ 2Department of Medical Informatics and Biostatistics, Iuliu Hațieganu University \\ of Medicine and Pharmacy, Cluj-Napoca, Romania \\ ${ }^{3}$ Department of Neurology, Iuliu Hațieganu University of Medicine and Pharmacy, \\ Cluj-Napoca, Romania \\ ${ }^{4}$ Department of Neurology, The University of Oklahoma Health Sciences Center \\ \& VA Medical Center, Oklahoma City, OK, USA
}

Submitted: 12 December 2015

Accepted: 22 April 2016

Arch Med Sci 2017; 13, 5: 1160-1167

DOI: $10.5114 /$ aoms.2016.60129

Copyright @ 2016 Termedia \& Banach

\section{Abstract}

Introduction: Cortical atrophy is known to be a valuable sign of cognitive decline. The purpose of this study was to assess the association between low thyroid-stimulating hormone (TSH), posterior cortical atrophy (Koedam score - KS) and nitro-oxidative stress in elderly patients.

Material and methods: A study (SG) and a control group (CG), each subdivided by gender, were investigated. Subjects older than 59 years with low serum TSH level and with mild cognitive impairment were included in the SG. The CG was formed by subjects free of significant cortical atrophy and free or thyroid dysfunction. Demographic and clinical characteristics of the patients (Mini Mental State Examination, MMSE), Koedam score on cranial magnetic resonance imaging, and blood parameters (TSH, FT4, and nitric oxide - NOx) were assessed.

Results: Subjects in the study group had fewer years of education above the 8 th grade compared with the control group ( $p<0.0001)$. A significantly higher percentage of subjects in the study group had a Koedam score of 2 or 3 compared with controls, who had in the majority of cases a Koedam score of zero $(p<0.02)$. Significantly higher NOx levels were observed when study groups of both genders were compared with corresponding controls $(p<0.001)$. No significant differences were observed with regard to FT4 $(p>0.70)$. Nitric oxide was found to be significantly associated with TSH $(p<0.03)$ and KS $(p<0.002)$ when the whole study group was considered as well as when just the non-smoker study group was investigated.

Conclusions: Our study revealed an association between subclinical thyroid hypofunction, nitro-oxidative stress, and posterior cortical atrophy as an early stage of global atrophy.

Key words: thyroid, cortical atrophy, oxidative stress, cognitive dysfunction.

\author{
Corresponding author: \\ Sorana D. Bolboacă PhD \\ Department of Medical \\ Informatics and Biostatistics \\ Iuliu Hațieganu University \\ of Medicine and Pharmacy \\ 6 Pasteur St \\ 400349 Cluj-Napoca, \\ Romania \\ Phone: +40 742609890, \\ +40264431697 \\ E-mail: sbolboaca@umfcluj.ro
}

\section{Introduction}

Subclinical hypothyroidism has a prevalence of $20 \%$, while the prevalence of hyperthyroidism is between 2 and $8 \%$ in the elderly population $[1,2]$. Diagnosis of thyroid dysfunction in the elderly population 
is a challenge. However, its diagnosis is possible due to the effects on the endothelium, heart, and brain function [3-5]. Inflammatory disorders (in chronic inflammatory diseases, a preponderance of energy expenditure pathways are switched on and thyroid hormones become energy expenditure hormones [6]) can influence the serum level of thyroid hormones in the elderly population. Furthermore, medication can also interfere with thyroid function tests [7].

Nitric oxide (NO) assessment can be a valuable biomarker for the amplitude of oxidative stress, being associated with both hyperthyroidism and hypothyroidism, as previously reported $[8,9]$. In clinical practice, both thyroid dysfunctions and cognitive impairment are more frequent in elderly women compared with elderly men [10], so the question is Are thyroid dysfunctions and cognitive impairment connected? One of these connections is emphasized by the beneficial effect of estrogen therapy on cognitive dysfunction in postmenopausal women [11]. However, the estrogen drugs should not be used as a preventive therapy for cognitive decline [12].

One of the most valuable signs associated with cognitive decline is cortical atrophy. Quantitative magnetic resonance imaging (MRI) is sensitive to structural changes associated with cortical atrophy. Evidence of structural changes may be found even in patients with mild cognitive impairment [13]. In the early phases of cognitive decline, the most prominent posterior brain regions are affected by atrophy, spreading to the anterior regions of the brain. The most affected region is the associative cortex, with relative sparing of sensorimotor regions [14].

The aim of this study was to explore the potential association between thyroid function (represented by low level of low thyroid-stimulating hormone (TSH)), oxidative stress (represented by serum level of NOx) and posterior cortical atrophy (the first stage of global cortical atrophy) in patients with cognitive dysfunctions. Furthermore, we sought to explore whether there are gender differences regarding the parameters above.

\section{Material and methods}

The study was conducted in accordance with the Helsinki Declaration and was approved by the local Ethics Committee (Clinical Rehabilitation Hospital, Cluj-Napoca, Romania). The informed consent form was signed by each subject included in the study. Subjects who referred to the Clinical Rehabilitation Hospital, Cluj-Napoca, Romania for neurological consultation during January 2012March 2015 were eligible for the study.

Patients older than 59 years with mild cognitive impairment and low TSH were included in the study group (SG). The control group (CG) was formed by subjects free of significant cortical atrophy and free or thyroid dysfunction who referred to the same hospital for neurological consultation. Both groups were subdivided by gender.

Because of numerous drug interferences with thyroid function (thyreostatic or thyromimetic medication), patients with any hormonal therapy, glucocorticoid medication and/or antipsychotic drugs were excluded from the study. Furthermore, patients with hypothyroidism, diabetes mellitus, and blood hypertension were also excluded from the sample. The presence of any cerebrovascular disease was also an exclusion criterion.

The groups were as follows:

- control group (CG, $n=182$ ) - patients without cognitive impairment and with normal thyroid function - further subdivided by gender as control group women (CW, $n=92)$ and control group men (CM, $n=90)$.

- study group (SG, $n=163$ ) - patients with mild cognitive impairment and low thyroid-stimulating hormone - further subdivided by gender as study group women (SW, $n=83$ ) and study group men (SM, $n=80)$.

All patients included in the study had neurological (because of cognitive impairment) consultation. The cognitive assessment was done with the Mini Mental State Examination (MMSE) test. The MMSE score classifies the cognitive impairment as follows: mild cognitive impairment (21-24 points), moderate cognitive impairment (10-20 points), and severe cognitive impairment (0-9 points). Spatial orientation (accounting for 5 points of the MMSE score) and visuospatial ability (accounting for 1 point of the MMSE score) were considered separately because of their association with posterior cortical atrophy $[15,16]$. This score, with a maximum of 6 points, was abbreviated as MMSE-PCA.

Blood samples were taken from all subjects included in the study in the morning (12 h fasting previous venous puncture for blood samples) and the samples were assessed in the Clinical Rehabilitation Hospital laboratory. The serum levels of TSH, FT4, NOx (nitrate and nitrite as a marker of nitro-oxidative stress) were determined. Total NOx levels were used as a surrogate marker for serum nitric oxide levels [17].

Cranial MRI examination was performed on all subjects included in the study for exclusion of cognitive impairment due to vascular etiology, and for assessment of cortical atrophy. The cranial MRI studies were performed with a $1.5 \mathrm{~T}$ device by assessing the morphologic features of the posterior parietal lobe. T1 and T2 weighted and Flair images were examined regarding axial, coronal and sagittal planes. The Koedam score (KS) was used to 
assess the posterior cortical atrophy. This score results from assessing multiple brain structures in three planes: sagittal (posterior cingulate sulcus, parieto-occipital sulcus, precuneus gyri), coronal (posterior cingulate sulcus, parietal gyri), and axial (posterior cingulate sulcus, parietal lobes) planes. The features were used to generate a grade from 0 to 3 [18]: grade 0: closed sulci, no gyral atrophy; grade 1: mild sulcal widening, mild gyral atrophy; grade 2: substantial sulcal widening, substantial gyral atrophy, and grade 3: marked sulcal widening, knife-blade gyral atrophy.

Several demographic characteristics such as age (years), body mass index (BMI, $\left.\mathrm{kg} / \mathrm{m}^{2}\right)$, education level (as years of education above $8^{\text {th }}$ grade), smoking status, and alcohol consumption (as occasional, moderate, or daily) were also recorded.

\section{Statistical analysis}

Demographic and baseline characteristics were summarized as median and the interquartile range (first and third quartiles) for quantitative data that proved not to follow the normal distribution (tested with the Anderson-Darling test). Qualitative data were summarized as percentages and associated $95 \%$ confidence intervals calculated using an exact formula [19]. Comparisons between groups were performed with the Mann-Whitney test for quantitative data and with the Z-test for qualitative data using two-sided $p$-values. Statistical analysis was conducted with Statistica software (v. 8, StatSoft) at a significance level of $5 \%$.
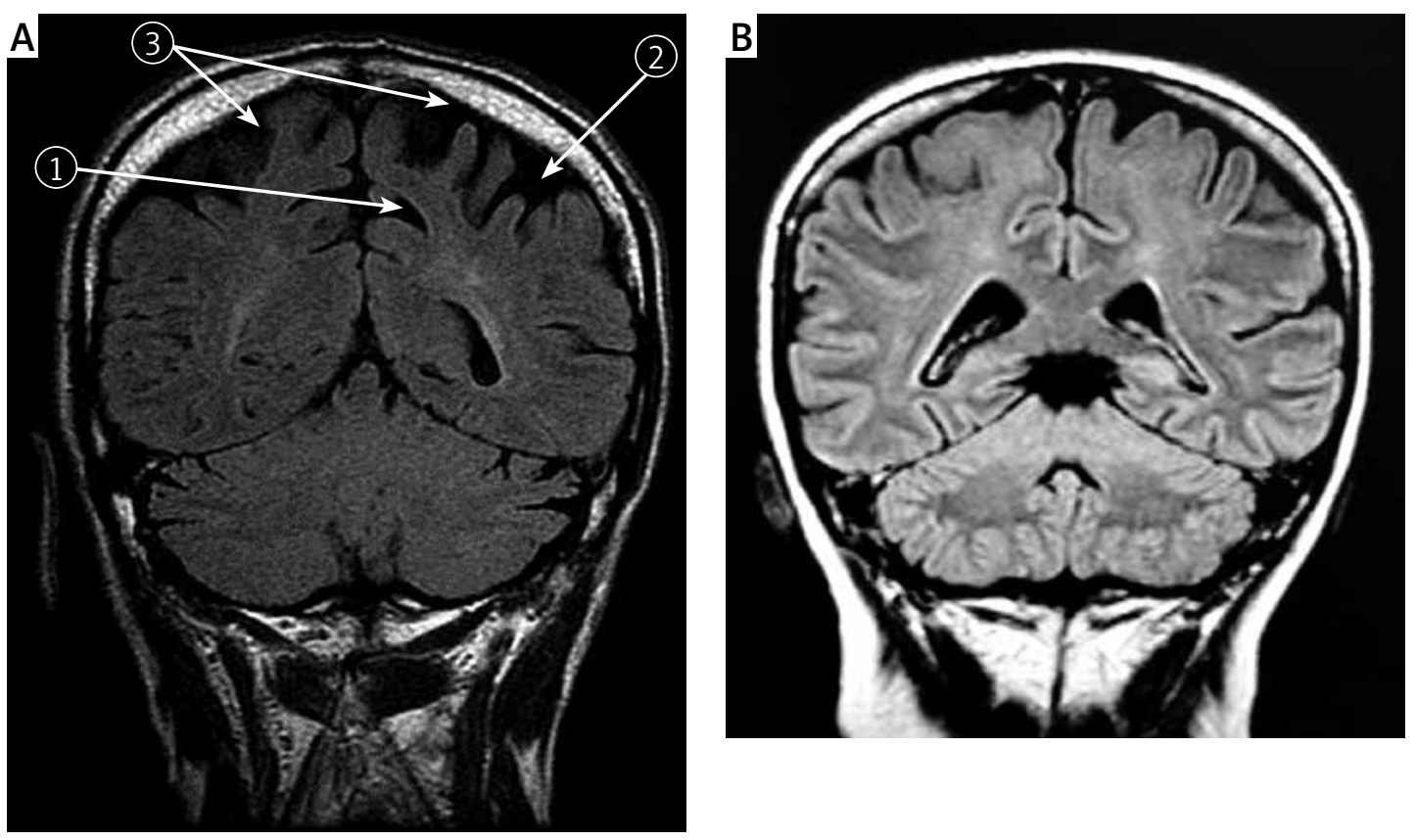

Figure 1. A - Cranial MRI on a patient in study group; FLAIR examination, coronal plane: (1) widening of posterior cingulate sulcus, (2) widening of intraparietal sulcus, (3) left and right superior parietal lobe atrophy (5, 7 Brodmann area); $\mathbf{B}$ - Cranial MRI on a patient in control group

The KS assessment (visual rating scale) on cranial MRI examination proved the presence of posterior cortical atrophy, as shown in Figure 1.

\section{Control vs. study group}

Demographic and clinical characteristics of the SG versus the CG are presented in Table I. Patients and control subjects did not differ in terms of gender or body mass index $(p>0.80)$. The age of subjects in the SG was significantly higher than in the CG group ( $p<0.001)$, and educational level showed significant differences between groups $(p<0.001)$.

All subjects in the SG had mild cognitive impairment (the values of MMSE between 20 and 24) while all subjects in the CG were normal with regard to cognitive impairment (statistically significant difference between groups with $p<0.001$ ). A higher percentage of subjects in the SG were also smokers compared to those from the CG $(p<0.0001)$.

The analysis conducted just on subjects who did not smoke showed no significant differences between case $(n=104)$ and control $(n=154)$ groups with regard to FT4 ( $p=0.9457)$. Moreover, the differences identified with regard to NOx, MMSE and KS in the whole sample were also identified in non-smoker samples $(p<0.0001)$.

\section{Control women vs. study group women}

Differences with regard to demographics and clinical characteristics between control group 
women (CW) and study group women (SW) are presented in Table II. As compared to the whole samples of CG and SG, with one exception, similar results were obtained in women. The exception is related to BMI, which proved significantly lower in SW compared to CW, but the values in both groups are in the normal range (see Table II).

The analysis conducted just on non-smoker women subjects revealed no significant differences between study $(n=58)$ and control $(n=82)$ group women with regard to FT4 $(p=0.8756)$. Moreover, the differences identified with regard to NOx, MMSE and KS in the whole women sample were also identified in samples that comprised just non-smoker women $(p<0.0001)$.

\section{Study group men vs. control group men}

Demographic and clinical characteristics of control group men (CM) and study group men (SM) are presented in Table III. Opposite to women, the BMI in male groups proved significantly higher for the group with PCA and TD compared to controls, but the values are also in the normal ranges (Table III).

The analysis was conducted just on subjects who did not smoke, and no significant difference between case $(n=46)$ and control $(n=72)$ groups was identified for FT4 $(p=0.9777)$. However, the differences identified with regard to NOx, MMSE and KS in the whole sample were also identified in samples that comprised just non-smokers $(p<0.0001)$.

\section{Study group women vs. study group men - gender differences}

The age of SM, slightly higher than SW, proved not significantly different at a significance level of $5 \%(p=0.0525)$.

Body mass index had higher values in the SM group compared with the SW group, but the values in both groups were in the normal ranges (the values in men were closest to the threshold of the overweight class; $p<0.0001)$. In the study group, no significant differences were observed when MMSE or MMSE-PCA was compared between gen$\operatorname{ders}(p>0.05)$.

The TSH values had significantly higher in the SM group compared with the SW group ( $p=0.0015)$, while the NOx value had significantly higher values in the SW group compared with the SM group $(p<0.0001)$.

A significantly higher number of men in the SM group had $\mathrm{KS}=1$ compared with women in the SW group $(p=0.0031)$. Conversely, a significant-

Table I. Demographic and clinical variables. Differences between patients (SG) and control group (CG)

\begin{tabular}{|c|c|c|c|}
\hline Variable & CG $(n=182)$ & SG $(n=163)$ & $P$-value \\
\hline Age [years] ${ }^{a}$ & $70(66-71)$ & $71(70-76)$ & $<0.0001$ \\
\hline BMI (Quetelet index $\left.\left[\mathrm{kg} / \mathrm{m}^{2}\right]\right)^{\mathrm{a}}$ & $24(23-24.5)$ & $24(23-24.5)$ & 0.8073 \\
\hline Further education ${ }^{\text {a }}$ (years of education above $8^{\text {th }}$ grade) & $7(4-8)$ & $4(3-4)$ & $<0.0001$ \\
\hline Current smokers (\%) ${ }^{\mathrm{b}}$ & $15.38[10.44-21.43]$ & $36.20[28.84-44.17]$ & $<0.0001$ \\
\hline \multicolumn{4}{|l|}{ Alcohol consumption (\%) } \\
\hline Occasional & $12.64[8.25-18.13]$ & $14.72[9.82-20.86]$ & 0.5743 \\
\hline Moderate & $6.04[2.75-10.44]$ & $5.52[2.46-10.43]$ & 0.8366 \\
\hline Daily & $1.10[0.003-3.84]$ & $2.45[0.62-6.13]$ & 0.3387 \\
\hline $\mathrm{FT} 4[\mathrm{ng} / \mathrm{dl}]^{\mathrm{a}}$ & $1.2(1.1-1.4)$ & $1.2(1.1-1.35)$ & 0.8166 \\
\hline $\mathrm{NOx}[\mu \mathrm{mol} / \mathrm{l}]^{\mathrm{a}}$ & $43.78(40.66-55.21)$ & $98(82-101.23)$ & $<0.0001$ \\
\hline MMSE [points] ${ }^{a}$ & $30[29-30]$ & $22[21-24]$ & $<0.0001$ \\
\hline MMSE-PCA [points] ${ }^{a}$ & $6[6-6]$ & $2[1-3]$ & $<0.0001$ \\
\hline \multicolumn{4}{|l|}{ KS [points] $]^{b}$} \\
\hline 0 & 95.05 [90.66-97.80] & $0.00[0.00-2.45]$ & $<0.0001$ \\
\hline 1 & $4.95[2.20-9.34]$ & $25.77[19.02-33.12]$ & $<0.0001$ \\
\hline 2 & $0.00[0.00-2.20]$ & $38.04[30.68-46.01]$ & $<0.0001$ \\
\hline 3 & $0.00[0.00-2.20]$ & $36.20[28.84-44.17]$ & $<0.0001$ \\
\hline
\end{tabular}

$B M I$ - body mass index, ${ }^{a}$ median (Q1-Q3), Q1 $=25^{\text {th }}$ percentile; $Q 3=75^{\text {th }}$ percentile; Mann-Whitney test. ${ }^{b} \%[95 \% \mathrm{Cl}], \mathrm{Cl}=\mathrm{confidence}$ interval [lower bound-upper bound]; $Z$ test for proportions. 
Table II. Demographic and clinic characteristics: differences between control group women (CW) and study group women (SW)

\begin{tabular}{|c|c|c|c|}
\hline Variable & $\mathrm{CW}(n=92)$ & SW $(n=83)$ & $P$-value \\
\hline Age [years] ${ }^{a}$ & $70(66-71)$ & $72(70-77)$ & $<0.0001$ \\
\hline BMI (Quetelet index $\left.\left[\mathrm{kg} / \mathrm{m}^{2}\right]\right)^{\mathrm{a}}$ & $24(23.5-24.5)$ & $23.8(22-24.5)$ & 0.0004 \\
\hline Further education ${ }^{\mathrm{a}}$ (years of education above $8^{\text {th }}$ grade) & $6(4-8)$ & $4(3-4)$ & $<0.0001$ \\
\hline Current smokers (\%) ${ }^{\mathrm{b}}$ & $10.87[5.45-19.55]$ & $30.12[20.50-40.95]$ & 0.0018 \\
\hline \multicolumn{4}{|l|}{ Alcohol consumption (\%) } \\
\hline Occasional & $7.61[3.27-15.21]$ & $16.87[9.65-26.49]$ & 0.0615 \\
\hline Moderate & $3.26[1.10-8.68]$ & $1.20[0.02-6.01]$ & 0.3635 \\
\hline Daily & $0.00[0.00-4.34]$ & $0.00[0.00-4.81]$ & n.a \\
\hline $\mathrm{FT} 4[\mathrm{ng} / \mathrm{dl}]^{\mathrm{a}}$ & $1.2(1.1-1.4)$ & $1.2(1.1-1.35)$ & 0.7285 \\
\hline $\mathrm{NOx}[\mu \mathrm{mol} / \mathrm{l}]^{\mathrm{a}}$ & $55.20(44.18-58.23)$ & $\begin{array}{c}101.23 \\
(100.46-102.33)\end{array}$ & $<0.0001$ \\
\hline MMSE [points] ${ }^{a}$ & 30 [29-30] & $21[20-24]$ & $<0.0001$ \\
\hline MMSE-PCA [points] $]^{a}$ & $6[6-6]$ & $2[1-3]$ & $<0.0001$ \\
\hline \multicolumn{4}{|l|}{$\mathrm{KS}^{\mathrm{b}}$ : } \\
\hline 0 & 95.65 [89.14-98.90] & $0.00[0.00-4.81]$ & $<0.0001$ \\
\hline 1 & $4.35[1.10-10.86]$ & $15.66[8.45-25.29]$ & 0.0125 \\
\hline 2 & $0.00[0.00-4.34]$ & $33.73[24.11-44.56]$ & $<0.0001$ \\
\hline 3 & $0.00[0.00-4.34]$ & $50.60[39.77-61.43]$ & $<0.0001$ \\
\hline
\end{tabular}

$B M I$ - body mass index; ${ }^{a}$ median (Q1-Q3), Q1 $=25^{\text {th }}$ percentile; $Q 3=75^{\text {th }}$ percentile; Mann-Whitney test; ${ }^{b} \%[95 \% \mathrm{Cl}], \mathrm{Cl}=\mathrm{Confidence}$ interval [lower bound-upper bound]; $Z$ test for proportions.

Table III. Differences in demographic and clinical variables between study men (SM) and control men (CM)

\begin{tabular}{|c|c|c|c|}
\hline Variable & CM $(n=90)$ & SM $(n=80)$ & $P$-value \\
\hline Age [years] ${ }^{a}$ & $70(67.25-73)$ & $71(70-73)$ & 0.0035 \\
\hline BMI (Quetelet index $\left.\left[\mathrm{kg} / \mathrm{m}^{2}\right]\right)^{\mathrm{a}}$ & $24(23-24.5)$ & $24.5(24-25)$ & 0.0002 \\
\hline Further education ${ }^{\mathrm{a}}$ (years of education above $8^{\text {th }}$ grade) & $7(4-8)$ & $4(3-6)$ & $<0.0001$ \\
\hline Current smokers (\%) ${ }^{\mathrm{b}}$ & $20.00[12.23-29.99]$ & $42.50[31.27-53.73]$ & 0.0018 \\
\hline \multicolumn{4}{|l|}{ Alcohol consumptions (\%) } \\
\hline Occasional & $17.78[10.01-26.65]$ & $12.50[6.27-21.23]$ & 0.3411 \\
\hline Moderate & $8.89[3.35-16.65]$ & $10.00[3.77-18.73]$ & 0.8049 \\
\hline Daily & $2.22[0.01-7.76]$ & $5.00[1.27-12.48]$ & 0.3282 \\
\hline $\mathrm{FT} 4[\mathrm{ng} / \mathrm{dll}]^{\mathrm{a}}$ & $1.2(1.1-1.38)$ & $1.2(1.1-1.33)$ & 0.9344 \\
\hline $\mathrm{NOx}[\mu \mathrm{mol} / \mathrm{l}]^{\mathrm{a}}$ & $41(39-42)$ & $81.5(80-85.25)$ & $<0.0001$ \\
\hline MMSE [points] ${ }^{a}$ & $30[29-30]$ & $23[21-24]$ & $<0.001$ \\
\hline MMSE-PCA [points] ${ }^{a}$ & $6[6-6]$ & $2[1-3]$ & $<0.0001$ \\
\hline \multicolumn{4}{|l|}{$\mathrm{KS}^{\mathrm{b}}:$} \\
\hline 0 & 94.44 [87.79-97.77] & $0.00[0.00-4.98]$ & $<0.0001$ \\
\hline 1 & $5.56[2.24-12.21]$ & $36.25[26.27-47.48]$ & $<0.0001$ \\
\hline 2 & $0[0.00-4.43]$ & $42.50[31.27-53.73]$ & $<0.0001$ \\
\hline 3 & $0[0.00-4.43]$ & $21.25[12.52-31.23]$ & $<0.0001$ \\
\hline
\end{tabular}

$B M I-$ body mass index, ${ }^{a}$ median $(Q 1-Q 3), Q 1=25^{\text {th }}$ percentile; Q3 $=75^{\text {th }}$ percentile; Mann-Whitney test; ${ }^{b} \%[95 \% \mathrm{Cl}], \mathrm{Cl}-$ confidence interval [lower bound-upper bound]; $Z$ test for proportions. 
Table IV. Significant monotonic relationship expressed as Spearman's rank correlation (rho) and associated $p$-values (in round brackets)

\begin{tabular}{|lcc|}
\hline Relationship & $\begin{array}{c}\text { All study group (SG) } \\
(n=163)\end{array}$ & $\begin{array}{c}\text { Non-smoker study group (SG) } \\
(n=104)\end{array}$ \\
\hline TSH and NOx & $-0.2647(0.0006)^{*}$ & $-0.2203(0.0246)$ \\
\hline TSH and KS & $-0.1039(0.1871)$ & $-0.1142(0.2483)$ \\
\hline NOx and MMSE & $-0.1733(0.0269)^{* *}$ & $-0.1846(0.0606)$ \\
\hline NOx and KS & $0.2824(0.0003)^{*}$ & $0.3193(0.0010)$ \\
\hline${ }^{*} p<0.001,{ }^{* *} p<0.05$. & &
\end{tabular}

ly higher number of women in the SW group had $\mathrm{KS}=3$ compared with the number of men in the $\mathrm{SM}$ group with $\mathrm{KS}=3(p=0.0001)$.

\section{Relationships between levels of TSH, NOx, MMSE, MMSE-PCA, and KS}

Significant monotonic relationships were identified when relations of investigated parameters were assessed in the SG. A significant negative relationship was found between TSH and NOx, and between NOx and MMSE (Table IV). No significant model was identified by multivariate analysis either for the SW or for the SM.

\section{Discussion}

The influence of education on cognitive decline has already been established, individuals with high education maintaining a higher level of cognitive function during the aging process [20]. Delay of the cognitive decline in subjects with higher education is based on the cognitive reserve hypothesis, the brain reserve or compensatory ability being higher in these individuals [21]. Higher education level delays the onset of accelerated cognitive decline, but once it begins, it is faster [22]. Our study evaluated only the relationship between educational level and cognitive status at the moment of examination, and did not evaluate the cognitive decline rate. Furthermore, our patients were assessed at the beginning of their cognitive decline, as is reflected by the MMSE scores (see Table I).

Our study showed that women had significantly low values of TSH compared with men, as previously reported by Wijsman et al. [23]. Wijsman et al. observed a significant higher frequency of subclinical thyroid dysfunction in women (73.8\%) compared with men (64.0\%). Subclinical thyroid diseases (elevated or suppressed thyroid-stimulating hormone with normal T3 and T4 levels) have already been reported as being associated with cognitive dysfunction and Alzheimer's disease [24]. A study which assessed the relationship between dementia and alteration thyroid function found a positive association between two demen- tia subtypes (vascular dementia and Alzheimer's disease dementia) and subclinical thyroid disorder (both subclinical hyper- and hypothyroidism) [25]. A possible explanation of the association between both subclinical hypothyroidism and cognitive decline is the presence of cardiovascular diseases and its risk factors as contributors to cognitive impairment in later life [26]. However, the nature of the relationship between these two pathologies has to be established.

Our results confirm the previous research data that showed the influence of gender in cognitive decline. Barnes et al. reported gender differences consisting of higher scores in women than men regarding episodic memory and perceptual speed, lower scores in semantic memory, and equivalent scores in working memory and visual-spatial ability [27]. There is a hypothesis that emphasizes the role of endogenous sex hormones in maintenance of cognitive function. Low estradiol level in elderly woman was reported as a factor that leads to the decline in global cognitive function and verbal memory [28].

Our study demonstrated a significant relation between TSH and NOx, which is a biomarker for endothelial dysfunction linked to cardiovascular disorders (see Table IV). Note that this significant relation is observed both in the whole sample and in the sub-sample that includes just non-smokers (see Table IV). While the presence of nitric oxide is a good predictor for the presence of the vasodilatation mechanism (endothelium-dependent vasodilator mechanism) [29], excessive production of nitric oxide can result in tissues damage [30]. These effects are preceded by upregulation of endothelial nitric oxide synthase (eNOS) that enhances nitrosative stress and, in cerebral capillaries, leads to cerebral microvascular degeneration, diminished brain mass, and cerebral functional deficits [31]. Immunohistochemical studies demonstrated increased activity of endothelial nitric oxide (eNOS) synthesis also in Alzheimer's diseases in brain capillaries that can lead to cortical atrophy [32]. An experimental model of hypothyroidism showed that there is increased production of NO in thyroid tissue. This is probably associ- 
ated with the mechanism of thyroid vasculature changes (vascular expansion) that occurs in hypothyroidism. The administration of N-nitro-L-arginine methyl ester (NAME) significantly improves NO synthesis [33]. Redondo et al. demonstrated that NO is a key mediator of neuronal cell death through a variety of mechanisms, including energy depletion-induced necrosis by inhibition of mitochondrial respiration, glutamate release and/or subsequent excitotoxicity [34].

In accordance with these results and with the results reported in our study, we can support a possible association between increased production of NOx, decreased level of TSH and posterior cortical atrophy (significant correlations between NOx and MMSE just for the whole sample, and between NOx and KS; see Table IV). Elevated NOx production was associated with obesity in women [32]. Despite the fact that our results established a significant increased NOx level in women compared with men (and in both groups compared to the control group), the BMIs of women and men did not show significant differences compared with the control group and are in the range of normal values. The statistically significant difference between increased NOx levels in women compared with men (higher in women) in our groups could be explained by the significant smoking status difference between these two groups. Smoking can decrease bioavailability of nitric oxide, and by this mechanism can favor vasoconstriction in the cerebral arteries [35]. Duriez et al. found that the gray matter (GM), white matter (WM), and hippocampus (HIP) volumes were modified by smoking in men, but not in women, the volume being lower in current smokers than in former or non-smokers [36]. Therefore, smoking seems to induce not only changes in thyroid function tests, such as a decrease in TSH and increase in thyroid hormones [37], but also brain structural changes. On the other hand, the significant correlation between increased NOx level and KS in our study groups (see Table IV) may be explained by the contribution of NO stress to posterior cortical atrophy. However, the observed correlation in the whole sample could be related to smoking, since a borderline correlation was observed in the non-smoker study group (see Table IV). The hippocampal neurons, the center of cognition associated with learning and memory, are very sensitive to oxidative stress action that can lead to neuronal degeneration [38]. Inducible nitric oxide synthase, which enhances the nitric oxide production, was found enhanced in hippocampal neurons in amnesic mild cognitive impairment and Alzheimer's disease patients [39]. Since there is a connection between thyroid hormones and increased oxidative stress [40], we can suppose that, in our study group, subclinical hypothyroidism may be related to posterior cortical atrophy and cognitive impairment. There are several potential explanations of lower TSH level in the elderly. The aging process could determine the reduction of thyrotropin-releasing hormone (TRH) secretion, related to destruction of hippocampus neurons, decreasing pituitary responsiveness, and, consequently, leading to a low level of TSH [41]. TRH concentration was found to be decreased in the hippocampus in subjects with Alzheimer's disease (AD) compared with normal elderly controls, being associated with phosphorylation of the tau protein, potentially involved in $A D$ pathogenesis [42].

The limits of this study are related to the difference in age of the control group, compared to the study groups, and to the invariable cerebrovascular changes related to the aging process. No other research studying the associations between parameters investigated in our research were identified in the scientific literature. More studies are needed to demonstrate the connection between subclinical thyroid disorders (hypo- or hyperfunction [43]) and the first stages of cognitive impairment in the elderly population. If these pathophysiological mechanisms are discovered, therapeutic schemas could be developed to delay the cognitive decline in elderly patients.

In conclusion, our study demonstrated the connection between subclinical thyroid hypofunction, nitro-oxidative stress, and posterior cortical atrophy as an early stage of global atrophy. The mechanisms involved in the observed relations needs future investigations. Our results should be considered as a support for indication of endocrinological evaluation of patients with posterior cortical atrophy and cognitive decline.

\section{Conflict of interest}

The authors declare no conflict of interest.

\section{References}

1. Rai GS, Gluck T, Luttrell S. Clinical presentation of hypothyroidism in older persons. J Am Geriatr Soc 1995; 43: 592-3.

2. Roberts LM, Pattison H, Roalfe A, et al. Is subclinical thyroid dysfunction in the elderly associated with depression or cognitive dysfunction? Ann Intern Med 2006; 145: 573-81.

3. Popławska-Kita A, Siewko K, Telejko B, et al. The changes in the endothelial function and haemostatic and inflammatory parameters in subclinical and overt hyperthyroidism. Int J Endocrinol 2013; 2013: 981638.

4. Cappola AR, Arnold AM, Wulczyn K, Carlson M, Robbins J, Psaty BM. Thyroid function in the euthyroid range and adverse outcomes in older adults. J Clin Endocrinol Metabol 2015; 100: 1088-96.

5. Villanueva I, Alva-Sánchez C, Pacheco-Rosado J. The role of thyroid hormones as inductors of oxidative stress 
and neurodegeneration. Oxid Med Cell Longevity 2013; 2013: 218145.

6. Straub RH. Interaction of the endocrine system with inflammation: a function of energy and volume regulation. Arthr Res Therapy 2014; 16: 203.

7. Kennedy RL, Griffiths H, Gray TA. Amiodarone and the thyroid. Clin Chem 1989; 35: 1882-7.

8. Dahiya K, Verma M, Dhankhar R, Singh V, Ghalaut PS, Seth S. Alteration of ischemia modified albumin and nitric oxide levels in hypothyroidism. Clin Labor 2014; 60: 969-72.

9. Makay O, Yenisey C, Icoz G, et al. The role of allopurinol on oxidative stress in experimental hyperthyroidism. J Endocrinol Investig 2009; 32: 641-6.

10. Li R, Cui J, Shen Y. Brain sex matters: estrogen in cognition and Alzheimer's disease. Mol Cell Endocrinol 2014 389: 13-21.

11. Petrone AB, Gatson JW, Simpkins JW, Reed MN. Nonfeminizing estrogens: a novel neuroprotective therapy. Mol Cell Endocrinol 2014; 389: 40-7.

12. O'Brien J, Jackson JW, Grodstein F, Blacker D, Weuve J. Postmenopausal hormone therapy is not associated with risk of all-cause dementia and Alzheimer's disease. Epidemiol Rev 2014; 36: 83-103.

13. McEvoy LK, Brewer JB. Quantitative structural MRI for early detection of Alzheimer's disease. Exp Rev Neu rother 2010; 10: 1675-88.

14. McDonald CR, McEvoy LK, Gharapetian L, et al.; Alzheimer's Disease Neuroimaging Initiative. Regional rates of neocortical atrophy from normal aging to early Alzheimer disease. Neurology 2009; 73: 457-65.

15. Herndon RM. Handbook of Neurologic Ranting Scale. Demos Medical Publishing, New York 2006; 201-2.

16. Gardini S, Concari L, Pagliara S, Ghetti C, Venneri A, Caffarra P. Visuo-spatial imagery impairment in posterior cortical atrophy: a cognitive and SPECT study. Behav Neurol 2011; 24: 123-32.

17. Giustarini D, Rossi R, Milzani A, Dalle-Donne I. Nitrite and nitrate measurement by Griess reagent in human plasma: evaluation of interferences and standardization. Methods Enzymol 2008; 440: 361-80.

18. Koedam EL, Lehmann M, van der Flier WM, et al. Visual assessment of posterior atrophy development of a MR rating scale. Eur Radiol 2011; 21: 2618-25.

19. Jäntschi L, Bolboacă SD. Exact probabilities and confidence limits for binomial samples: applied to the difference between two proportions. Sci World J 2010; 10: 865-78.

20. Stern Y, Gurland B, Tatemichi TK, Tang MX, Wilder D, Mayeux R. Influence of education and occupation on the incidence of Alzheimer's disease. JAMA 1994; 271: 1004-10.

21. Stern Y, Albert S, Tang MX, Tsai WY. Rate of memory decline in $A D$ is related to education and occupation: cognitive reserve? Neurology 1999; 53: 1942-7.

22. Hall CB, Derby C, LeValley A, Katz MJ, Verghese J, Lipton RB. Education delays accelerated decline on a memory test in persons who develop dementia. Neurology 2007; 69: 1657-64.

23. Wijsman LW, de Craen AJ, Trompet S, et al. Subclinical thyroid dysfunction and cognitive decline in old age. PLoS One 2013; 8: e59199.

24. Volpato S, Guralnik JM, Fried LP, Remaley AT, Cappola AR, Launer LJ. Serum thyroxine level and cognitive decline in euthyroid older women. Neurology 2002; 58: 1055-61.

25. Benseñor I, Lotufo PA, Menezes PR, Scazufca M. Subclinical hyperthyroidism and dementia: the Sao Paulo Ageing \& Health Study (SPAH). BMC Public Health 2010; 10: 298.
26. O'Brien JT, Markus HS. Vascular risk factors and Alzheimer's disease. BMC Medicine 2014; 12: 218.

27. Barnes LL, Wilson RS, Schneider JA, Bienias JL, Evans DA, Bennett DA. Gender, cognitive decline, and risk of AD in older persons. Neurology 2003; 60: 1777-81.

28. Yaffe K, Barnes D, Lindquist K, et al.; Health ABC Investigators. Endogenous sex hormone levels and risk of cognitive decline in an older biracial cohort. Neurobiol Aging 2007; 28: 171-8.

29. Verma S, Anderson TJ. Fundamentals of endothelial function for the clinical cardiologist. Circulation 2002; 105: 546-9.

30. Kertmen H, Gürer B, Yilmaz ER, et al. Antioxidant and antiapoptotic effects of darbepoetin against traumatic brain injury in rats. Arch Med Sci 2015; 11: 1119-28.

31. Sirinyan M, Sennlaub F, Dorfman A, et al. Hyperoxic exposure leads to nitrative stress and ensuing microvascular degeneration and diminished brain mass and function in the immature subject. Stroke 2006; 37: 2807-15.

32. Provias J, Jeynes B. Correlation analysis of capillary APOE, VEGF and eNOS expression in Alzheimer brains. Curr Alzheimer Res 2011; 8: 197-202.

33. Colin IM, Nava E, Toussaint D, et al. Expression of nitric oxide synthase isoforms in the thyroid gland: evidence for a role of nitric oxide in vascular control during goiter formation. Endocrinology 1995; 136: 5283-90.

34. Redondo J, Hares K, Wilkins A, Scolding N, Kemp K. Reductions in kinesin expression are associated with nitric oxide-induced axonal damage. J Neurosci Res 2015; 93 : 882-92.

35. Koide $M$, Nishizawa S, Yamamoto S, Yamaguchi $M$, Namba H, Terakawa S. Nicotine exposure, mimicked smoking, directly and indirectly enhanced protein kinase $C$ activity in isolated canine basilar artery, resulting in enhancement of arterial contraction. J Cerebral Blood Flow Metabol 2005; 25: 292-301.

36. Duriez Q, Crivello F, Mazoyer B. Sex-related and tissue-specific effects of tobacco smoking on brain atrophy: assessment in a large longitudinal cohort of healthy elderly. Front Aging Neurosci 2014; 6: 299.

37. Sawicka-Gutaj N, Gutaj P, Sowiński J, et al. Influence of cigarette smoking on thyroid gland - an update. Endokrynol Pol 2014; 65: 54-62.

38. Daulatzai MA. Neurotoxic saboteurs: straws that break the hippo's (hippocampus) back drive cognitive impairment and Alzheimer's disease. Neurotox Res 2013; 24: 407-59.

39. Barone E, Di Domenico F, Cenini G, et al. Oxidative and nitrosative modifications of biliverdin reductase- $A$ in the brain of subjects with Alzheimer's disease and amnestic mild cognitive impairment. J Alzheimer's Dis 2011; 25: 623-33.

40. Bianchi G, Solaroli E, Zaccheroni V, et al. Oxidative stress and anti-oxidant metabolites in patients with hyperthyroidism: effect of treatment. Horm Metab Res 1999; 31 : 620-4.

41. van Osch LA, Hogervorst E, Combrinck M, Smith AD. Low thyroid-stimulating hormone as an independent risk factor for Alzheimer disease. Neurology 2004; 62: 1967-71.

42. Luo L, Yano N, Mao Q, Jackson IM, Stopa EG. Thyrotropin releasing hormone (TRH) in the hippocampus of Alzheimer patients. J Alzheimer's Dis 2002; 4: 97-103.

43. Kang C. Using ultrasound radio frequency technology to assess regression of the structure and function of the carotid artery by radioiodine therapy in hyperthyroidism patients. Arch Med Sci 2015; 11: 1236-43. 